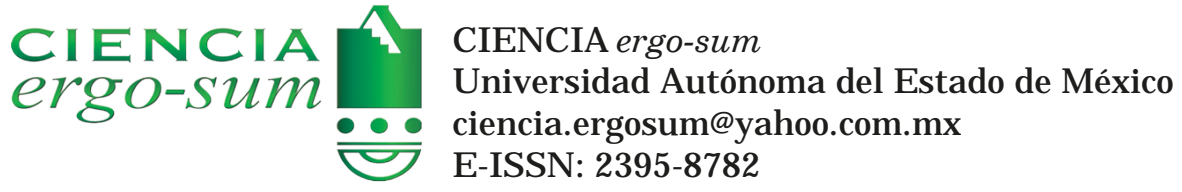

\title{
Distribución de Mammuthus columbi (Mammalia, Proboscidea) en el Pleistoceno tardío de Puebla, México
}

Lagunas Rodríguez, Zaid; Carbot-Chanona, Gerardo; J iménez Moreno, Francisco J avier

Distribución de Mammuthus columbi (Mammalia, Proboscidea) en el Pleistoceno tardío de Puebla, México

CIENCIA ergo-sum, vol. 26, núm. 2, julio-octubre 2019| e53

Universidad Autónoma del Estado de México, México

Esta obra está bajo una Licencia Creative Commons Atribución-NoComercial-SinDerivar 4.0 Internacional.

Lagunas Rodríguez, Z., Carbot-Chanona, G. y J iménez Moreno, F. J . (2019). Distribución de Mammuthus columbi (Mammalia, Proboscidea) en el Pleistoceno tardío de Puebla, México. CIE N CIA ergo-sum, 26(2). https:/l doi.org/10.30878/ces.v26n2a7 


\title{
Distribución de Mammuthus columbi (Mammalia, Proboscidea) en el Pleistoceno tardío de Puebla, México
}

Distribution of Mammuthus columbi (Mammalia, Proboscidea) in the Late Pleistocene of Puebla, Mexico

\author{
Zaid Lagunas Rodriguez \\ Centro INAH-Puebla, México \\ zaidlagunas@yahoo.com.mx \\ Gerardo Carbot-Chanona \\ Museo de Paleontología "Eliseo Palacios Aguilera", México \\ gfcarbot@gmail.com \\ Francisco Javier Jiménez Moreno \\ Posgrado en Ciencias Ambientales, BUAP, México \\ pacosaurus1@gmail.com
}

Recepción: 31 de enero de 2017

\section{RESUMeN}

Se hace un recuento de las localidades con restos de Mammuthus columbi en Puebla. Los resultados arrojan 32 localidades previamente reportadas y seis nuevas, lo cual representa $14 \%$ del total de las que se habían reportado para México, y ubican a Puebla como el segundo estado con más restos detrás del Estado de México. Por otro lado, la distribución de las localidades corresponde con el corredor biogeográfico Eje Neovolcánico Transversal-Sierra Madre del Sur. La amplia distribución de restos de Mammuthus columbi demuestra el potencial con el que cuenta el estado y da un punto de partida para futuras exploraciones en pro de ampliar el conocimiento sobre el pasado de México.

Palabras clave: Mammuthus columbi, paleodistribución, paleoambiente, Puebla.

\begin{abstract}
We made a recount of the localities with Mammuthus columbi remains in Puebla State. Our results show 32 previously reported localities and six new ones, they are distributed in the central-western area of the State between the Sierra Norte of Puebla and the Mixteca Poblana, and an altitude from 1500 to 2500 meters above sea level. This represents the $14 \%$ of the total of localities in Mexico and places Puebla as the second state with the most Mammuthus fossil remains, behind the State of Mexico. On the other hand, the distribution of the localities corresponds to the Transvolcanic Belt-Sierra Madre del Sur biogeographic corridor. On the other hand, the extensive distribution of M. columbi remains demonstrate the fossiliferous potential of the state, and gives a starting point for future explorations in order to enhance knowledge on the past of Mexico.
\end{abstract}

KEYWORDs: Mammuthus columbi, paleodistribution, paleoenvironment, Puebla.

\section{INTRODUCCIÓN}

El Pleistoceno fue una época dinámica con marcados cambios climáticos caracterizada por periodos fríos y el avance de glaciares, denominados glaciaciones, intercalados por periodos cálidos o interglaciares, lo que incidió en la distribución, especiación, dispersión y extinción de varios grupos biológicos existentes (Graham, 1998). El Pleistoceno posee cuatro divisiones globales: Gelasiano (2.58-1.8 Ma), Calabriano (1.80-0.773 Ma), Pleistoceno Medio (0.773-0.126 Ma) y Pleistoceno Superior (0.126-0.0117 Ma) (Ogg et al., 2016). Esta época también se 
distinguió por las asociaciones de vertebrados, donde se mezclaban especies de afinidad neártica con especies de afinidad tropical; son asociaciones no análogas que no se presentan en la actualidad (Lundelius et al., 1987).

En México se conocen 776 localidades fosilíferas de edad pleistocénica, de las cuales $45 \%$ se ubica en el Eje Neovolcánico Transversal. Los estados con mayor cantidad de localidades son el Estado de México con 145 (18.69\%), Distrito Federal (hoy Ciudad de México) con 70 (9.02\%) y Puebla con 57 (7.35\%) (Arroyo-Cabrales et al., 2002). En casi todas las localidades se han reportado restos de megamamíferos, entre los que se cuenta el proboscídeo Mammuthus columbi (Arroyo-Cabrales et al., 2007a).

Mammuthus columbi es uno de los megamamíferos más distribuidos en América del Norte (Kurtén y Anderson, 1980), donde la región de Orotina, en Costa Rica, posee el registro más austral de esta especie en el continente (Laurito y Aguilar, 2007). En México M. columbi se distribuyó en casi todo su territorio; sin embargo, se desconoce su presencia en la península de Yucatán y Tabasco (Arroyo-Cabrales et al., 2010). El registro más sureño lo tiene la localidad El Amate, en Villaflores, Chiapas (Gómez-Pérez y Carbot-Chanona, 2012).

En el estado de Puebla se conocen diversas localidades del Pleistoceno tardío en donde hay registro de restos de Mammuthus (Lagunas Rodríguez y Suárez Cruz, 1997; Suárez Cruz y Lagunas Rodríguez, 2008; Lagunas Rodríguez et al., 2013; Jiménez-Hidalgo et al., 2015). No obstante, no todos los hallazgos son registrados o estudiados por especialistas y con ello se pierde valiosa información científica. Adicionalmente, a la fecha no existen trabajos que compilen todos los registros que se tienen para el estado. Por tal motivo, este artículo tiene como objetivo hacer un recuento de las localidades con restos de Mammuthus columbi en Puebla, adicionar nuevas localidades y comentar su paleodistribución en el estado.

\section{Registros históricos De mammuthus EN PUEBLA}

\section{1. Descubrimientos del siglo XV al XIX}

Los primeros reportes de Mammuthus se dan en el Códice Mendocino, donde son referidos como los gigantes Quinametli o Quinametzin (Torquemada, 1969); estos gigantes eran nombrados Xantilometl (huesos de gigante) por los habitantes de la región de Puebla (Armenta Camacho, 1978). A principios del siglo XIX, comienza su estudio y catalogación formal, lo cual pone fin a los mitos de gigantes (Arroyo-Cabrales et al., 2003b). En ese mismo siglo se forma la primera colección de fósiles que contenía restos de Mammuthus de la región Valsequillo, la cual se alojaba en el Gabinete de Historia Natural del antiguo Colegio del Estado, actualmente Benemérita Universidad Autónoma de Puebla (BUAP) (Armenta Camacho, 1978). A finales del siglo XIX, Felix y Lenk (1891) reportan molares referidos como Elephas primigenius var. columbi (= Mammuthus columbi) de la región de Tecamachalco y San Pedro.

\subsection{Descubrimientos en el siglo $X X$}

Entre los primeros trabajos del siglo XX destacan los de Osborn $(1905,1942)$, quien reconoce, basado en molares aislados, la presencia de Elephas imperator y Archidiskodon imperator en el Valle de Puebla, Elephas columbi var. felicis en Tecamachalco y Paralephas columbi en San Francisco Totimehuacán. Esta localidad también es referida por Pichardo del Barrio (1960). Posteriormente, Freudenberg (1922) reporta a Elephas columbi cf. var. felicis también para el Valle de Puebla.

Irwin-Williams (1967) publicó un trabajo donde proporciona información sobre los registros de Mammuthus en la zona de Valsequillo y Silva-Bárcenas (1969) menciona la presencia de Mammuthus imperator en la localidad San Pedro Zacachimalpa. Años más tarde, Armenta Camacho (1978) señala la presencia de restos fósiles de proboscídeos en la ribera del río Álseseca y de Mammuthus en la ciudad de Puebla, material que fue 
depositado en la Colección Osteológica del Departamento de Antropología de la Universidad de Puebla (CODAUP), hoy desaparecida.

Lagunas Rodríguez y Suárez Cruz (1997) rescataron restos de Mammuthus sp. en la colonia Tres Cerritos, ubicada al sur de la ciudad, que fueron asignados a Mammuthus imperator (= M. columbi).

\subsection{Descubrimientos en el siglo XXI}

El Instituto Nacional de Antropología e Historia, Centro INAH-Puebla, resguarda en la sección de Paleontología un total de 2243 ejemplares de vertebrados catalogados (Cruz-Muñoz, 2001), entre los que se encuentran restos de Mammuthus columbi. Muchos de estos restos proceden de diversas localidades de Puebla, producto del trabajo de rescate y conservación de los investigadores del Centro INAH-Puebla, de 1994 a la fecha. Entre los lugares donde se han rescatado restos de M. columbi están los municipios de Quecholac, Amozoc, Chalchicomula de Sesma y Santa Ana Teloxtoc (tabla 1).

Entre los reportes más recientes se incluye el de Tovar et al. (2014), quienes mencionan una importante asociación de micro y macrovertebrados fósiles en la localidad de Santa Cruz Nuevo, ubicada al sur del estado de Puebla, donde también se encontraron restos de Mammuthus sp.

\section{Material y MÉtodo}

Se compilaron los registros históricos y actuales usando información publicada e inédita sobre los registros de Mammuthus en el estado de Puebla. Esa información está basada en material rescatado y resguardado en la sección de Paleontología del Centro INAH-Puebla, del Instituto Nacional de Antropología e Historia, catalogado bajo el nombre de CRINAHP (Colección Regional del Instituto Nacional de Antropología e Historia, Puebla), y en la colección paleontológica de la Facultad de Ciencias Biológicas, Benemérita Universidad Autónoma de Puebla, catalogado bajo las siglas BUAPPALZ. Los ejemplares fueron determinados taxonómicamente basados en los caracteres diagnósticos de molares y elementos apendiculares. Los restos que no pudieron identificarse no fueron incluidos en este trabajo.

El mapa de distribución se elaboró con ArcMap 10.3 usando como capa base el mapa hipsométrico alojado en el portal de geoinformación de la Comisión Nacional para el Conocimiento y Uso de la Biodiversidad (CONABIO). Posteriormente, se modificó en CorelDraw X8 (versión 18).

\section{Resultados}

La revisión bibliográfica arrojó 32 localidades ya reportadas con restos de Mammuthus en Puebla (Armenta Camacho, 1978; Lagunas Rodríguez y Suárez Cruz, 1997; Cruz Muñoz, 2001; Arroyo-Cabrales et al., 2003b; Suárez Cruz y Lagunas Rodríguez, 2008; Lagunas Rodríguez et al., 2013). En particular, este artículo adiciona seis nuevas localidades dando un total de 38 (tabla 1). La mayoría de los sitios se ubican en la porción centro-occidental del estado, con algunas localidades al sur y dos al norte. Esta distribución tiene correspondencia con la altitud, ya que todas están ubicadas entre los 1500 a 2500 msnm y se ubican principalmente en el Eje Neovolcánico y Sierra Madre del sur (mapa 1).

La mayoría de los restos de Mammuthus procedentes de Puebla reportados en la bibliografía están desaparecidos o su repositorio es desconocido. Sin embargo, se pudo compilar un listado de 69 ejemplares procedentes del estado de Puebla y que están depositados en las colecciones paleontológicas del Centro INAH-Puebla y la Benemérita Universidad Autónoma de Puebla, así como en dos colecciones particulares (anexo 1). El laboratorio de arqueozoología del INAH, México también resguarda restos de Mammuthus del estado de Puebla. Sin embargo, no fue posible obtener el listado detallado de dicha colección. 
TABLA 1

Localidades con restos de Mammuthus columbi en Puebla

\begin{tabular}{|c|c|c|}
\hline $\begin{array}{l}\text { Número de } \\
\text { localidad }\end{array}$ & Localidad y municipio & Fuente \\
\hline 1 & San Antonio Arenillas, Valsequillo, Puebla & $\begin{array}{l}\text { Guenther y Bunde (1973), Armenta Camacho } \\
\text { (1978), Pichardo (1997), Arroyo-Cabrales et al. } \\
\text { (2003b) }\end{array}$ \\
\hline 2 & Barranca Caulapan, Valsequillo, Puebla & $\begin{array}{l}\text { Guenther y Bunde (1973), Pichardo (1997), Arro- } \\
\text { yo-Cabrales et al. (2003b) }\end{array}$ \\
\hline 3 & $\begin{array}{l}\text { Centro Histórico, ciudad de Puebla ( } 5 \text { de mayo y } 2 \\
\text { poniente) }\end{array}$ & Armenta (1978) \\
\hline 4 & Ixtacamaxtitlán & Cruz Muñoz (2001) \\
\hline 5 & Lagunillas de Rayón & Cruz Muñoz (2001) \\
\hline 6 & $\begin{array}{l}\text { Nopalucan de la Granja, barranca El Garzón y barran- } \\
\text { ca Zurcada Larga }\end{array}$ & Suárez Cruz y Lagunas Rodríguez (2008) \\
\hline 7 & Ocotitlán & Cruz Muñoz (2001) \\
\hline 8 & San Francisco Totimehuacán & $\begin{array}{l}\text { Osborn (1905), Pichardo del Barrio (1960), } \\
\text { Guenther y Bunde (1973), Pichardo (1997), } \\
\text { Arroyo-Cabrales et al. (2003b), Suárez Cruz y } \\
\text { Lagunas Rodríguez (2008) }\end{array}$ \\
\hline 9 & Colonia Tres Cerritos (ciudad de Puebla) & Suárez Cruz y Lagunas Rodríguez (2008) \\
\hline 10 & San Pedro Zacachimalpa & $\begin{array}{l}\text { Silva Bárcenas (1969), Guenther y Bunde (1973), } \\
\text { Pichardo (1997), Arroyo-Cabrales et al. (2003b) }\end{array}$ \\
\hline 11 & San Baltazar Tetela, Puebla & $\begin{array}{l}\text { Pichardo del Barrio (1960), Pichardo (1997), } \\
\text { Arroyo-Cabrales et al. (2003b) }\end{array}$ \\
\hline 12 & Tepexi de Rodríguez & Arroyo-Cabrales et al. (2003b) \\
\hline 13 & Santa Cruz Nuevo & Tovar et al. (2014) \\
\hline 14 & Cerro Puilco, Tecamachalco & Arroyo-Cabrales et al. (2003b) \\
\hline 15 & Valsequillo & $\begin{array}{l}\text { Guenther (1968), Guenther y Bunde (1973), Arro- } \\
\text { yo-Cabrales et al. (2003b) }\end{array}$ \\
\hline 16 & Acajete & Lagunas Rodríguez et al. (2013) \\
\hline 17 & Atlixco & Arroyo-Cabrales et al. (2003b) \\
\hline 18 & Tecamachalco (San Pedro) & $\begin{array}{l}\text { Felix y Lenk (1891), Arroyo-Cabrales et al. } \\
\text { (2003b) }\end{array}$ \\
\hline 19 & Santa Catarina Villanueva, Quecholac & $\begin{array}{l}\text { Suárez Cruz y Lagunas Rodríguez (2008), } \\
\text { Lagunas Rodríguez et al. (2013) }\end{array}$ \\
\hline 20 & Cuautinchána & Este trabajob \\
\hline 21 & $\begin{array}{l}\text { Amozoc (colonia Tres Cruces, Sierra de Amozoc, } \\
\text { camino a Cuautinchán) }\end{array}$ & Este trabajo \\
\hline 22 & Santo Tomás Chautla (Valsequillo) & $\begin{array}{l}\text { Suárez Cruz y Lagunas Rodríguez (2008), } \\
\text { Lagunas Rodríguez et al. (2013) }\end{array}$ \\
\hline 23 & San Andrés Cholula & Lagunas Rodríguez et al. (2013) \\
\hline 24 & San Lorenzo Almecatla & $\begin{array}{l}\text { Suárez Cruz y Lagunas Rodríguez (2008), } \\
\text { Lagunas Rodríguez et al. (2013) }\end{array}$ \\
\hline 25 & Cerro Chiquihuite, San Francisco Totimehuacán & Este trabajo \\
\hline
\end{tabular}


TABLA 1 (continúa)

\begin{tabular}{|c|c|c|}
\hline 26 & Canal de Valsequillo & Arroyo-Cabrales et al. (2003b) \\
\hline 27 & Santa Ana Teloxtoc, Tehuacán & Lagunas Rodríguez et al. (2013) \\
\hline 28 & $\begin{array}{l}\text { Barranca El Morillo, Ciudad Serdán, Chalchicomula } \\
\text { de Sesma }\end{array}$ & Lagunas Rodríguez et al. (2013) \\
\hline 29 & Hueyatlaco & $\begin{array}{l}\text { Guenther y Bunde (1973), Pichardo (1997), } \\
\text { Arroyo-Cabrales et al. (2003b) }\end{array}$ \\
\hline 30 & Atepetzingo & $\begin{array}{l}\text { Pichardo del Barrio (1960); Guenther y Bunde } \\
\text { (1973); Arroyo-Cabrales et al. (2003b) }\end{array}$ \\
\hline 31 & Tlatlauquitepec & Arroyo-Cabrales et al. $(2003 \mathrm{~b})^{\mathrm{c}}$ \\
\hline 32 & Rancho Gerardo, Tepexi de Rodríguez & Torres-Martínez y Agenbroad (1991) \\
\hline 33 & Colina del Gliptodonte, Tepexi de Rodríguez & Montellano-Ballesteros (2000) \\
\hline 34 & Barrio de San José Acatzingo & Este trabajo \\
\hline 35 & $\begin{array}{l}\text { Barranca La Presa, Ciudad Serdán (cerca de Barranca } \\
\text { El Morillo) }\end{array}$ & Este trabajo \\
\hline 36 & Ciudad de Puebla (4 poniente, número 306) & Armenta Camacho (1978) \\
\hline 37 & $\begin{array}{l}\text { Arroyo San Francisco, Ciudad de Puebla (hoy Boule- } \\
\text { vard } 5 \text { de mayo) }\end{array}$ & Armenta Camacho (1978) \\
\hline 38 & Atexcal & Este trabajo \\
\hline
\end{tabular}

\section{Discusión}

\section{1. Identidad taxonómica}

Dos especies dentro del género Mammuthus son reconocidas para el Pleistoceno de México, el llamado complejo $M$. hayi/meridionalis y $M$. columbi, especies que han sido diferenciadas por su morfología dental (Arroyo-Cabrales et al., 2003a). Mammuthus hayi/meridionalis cuenta con registros únicamente en sedimentos del Irvingtoniano en Arizpe, Sonora; El Mezquital, Baja California, y Culhuacán, Ciudad de México; sin embargo, Arroyo-Cabrales et al. (2007) cuestionan la identificación de dicho material. Por su parte, Lister y Sher (2015) examinaron el material asignado a M. hayi/meridionalis procedente de El Mezquital y Culhuacán y concluyen que las medidas y frecuencia lamelar caen en el rango de $M$. columbi, por lo que argumentan que M. hayi/meridionalis no estuvo presente en México. En consecuencia, siguiendo a Lister y Sher (2015), consideramos que la única especie presente en el Pleistoceno de México es M. columbi.

M. columbi generalmente presenta molares grandes y angostos, con láminas de gruesas a delgadas, esmalte fuertemente crenulado y una frecuencia lamelar de 5 a 7 (Arroyo-Cabrales et al., 2003a). El material dental de Mammuthus alojado en el Centro INAH-Puebla y en la colección paleontológica de la BUAP posee las características morfológicas de $M$. columbi, por lo que son asignados a esta especie.

Por su parte, el material poscraneal aislado que no fue recuperado en asociación a restos dentales se asigna a Mammuthus columbi y no a Cuvieronius o Mammut, ya que los huesos apendiculares de estos dos últimos géneros son más cortos y masivos que en Mammuthus (Olsen, 1972; Tapía-Ramírez et al., 2013; Bravo-Cuevas et al., 2015). 


\section{2. Paleodistribución}

Basado en los reportes existentes, en el mapa 1 se observa que Mammuthus columbi estuvo distribuido en su mayoría en el centro del estado de Puebla, en sitios que oscilan entre los 1500 y 2500 msnm, y habitó en las cuencas formadas entre la Sierra Norte de Puebla y la mixteca poblana. Es probable que estas cuencas fueran parte del corredor biogeográfico Eje Neovolcánico Transversal-Sierra Madre del Sur (mapa 2), una de las siete rutas usadas por los mamíferos para dispersarse a través de México (sensu Ceballos et al., 2010).

Aunque la mayoría de los registros carecen de control estratigráfico, muchos de los ejemplares vienen de depósitos en donde se han recuperado también restos de Bison sp., por lo que pueden ser referidos a la edad rancholabreana dentro de los NALMA (North American Land Mammal Ages, Pleistoceno Tardío), que abarca de 160000 a 9500 años (Bell et al., 2004). Los únicos restos que cuentan con datación absoluta por radiocarbono proceden de la Cuenca de Valsequillo, donde se han identificado tres niveles fosilíferos: la zona I, datada en 9 $150 \pm 500$ años, que incluye restos asignados a M. felicis (= M. columbi) de las localidades Caulapan y Tecamachalco; la zona II, datada entre $26000 \pm 530$ y $21000 \pm 1500$ años, con restos de M. columbi de las localidades Zacachimalpa, Hueyatlaco, Atepetzingo, Caulapan y Arenillas; la zona III, con la presencia de M. imperator (= M. columbi) en las localidades de Arenillas, Totimehuacán y Caulapan (Pichardo, 1997).

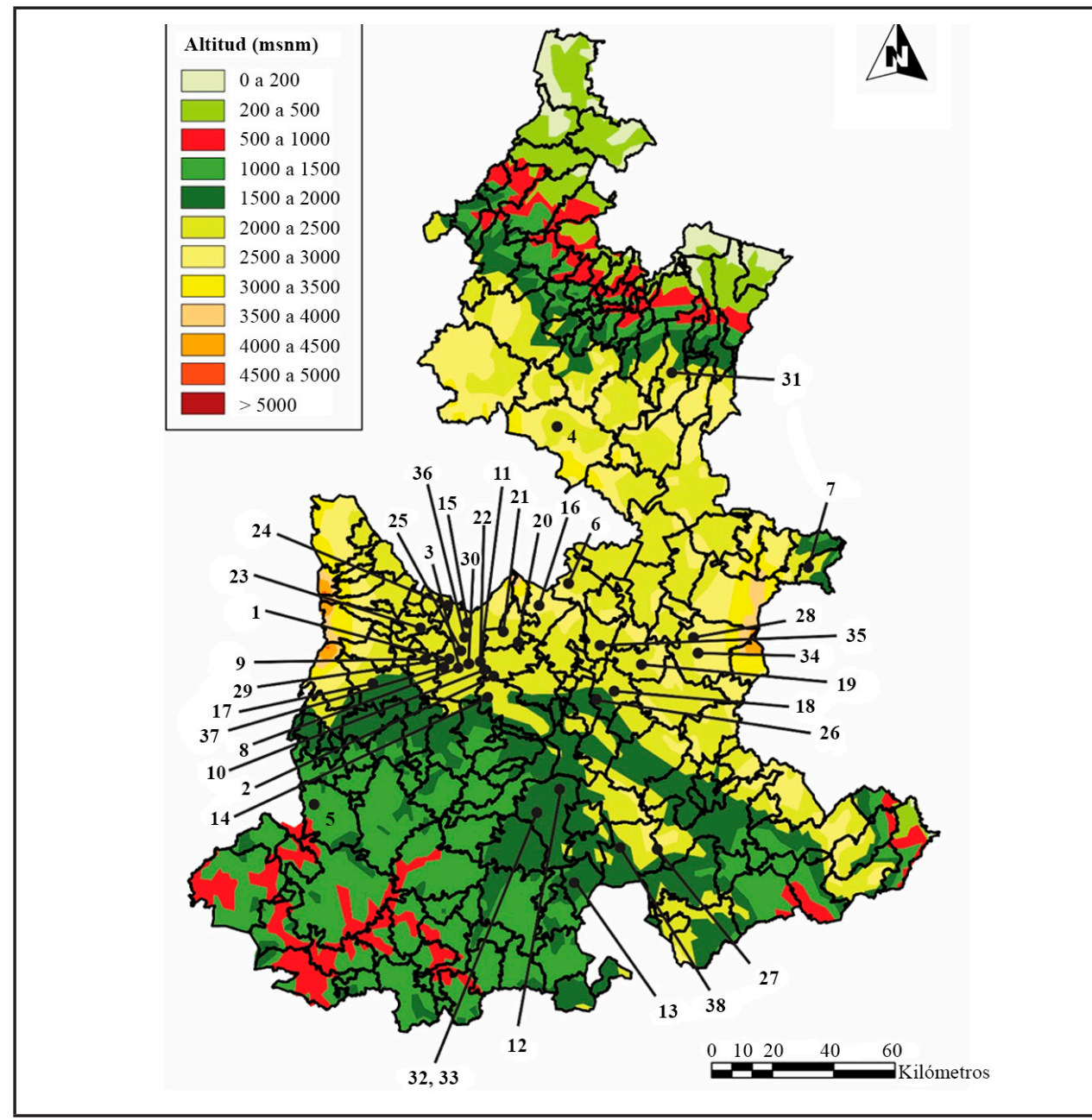

MAPA 1

Mapa hipsométrico que muestra las localidades fosilíferas con presencia de Mammuthus columbi en Puebla, México

Fuente: elaboración propia.

Nota: la numeración corresponde con las localidades plasmadas en la tabla 1. 


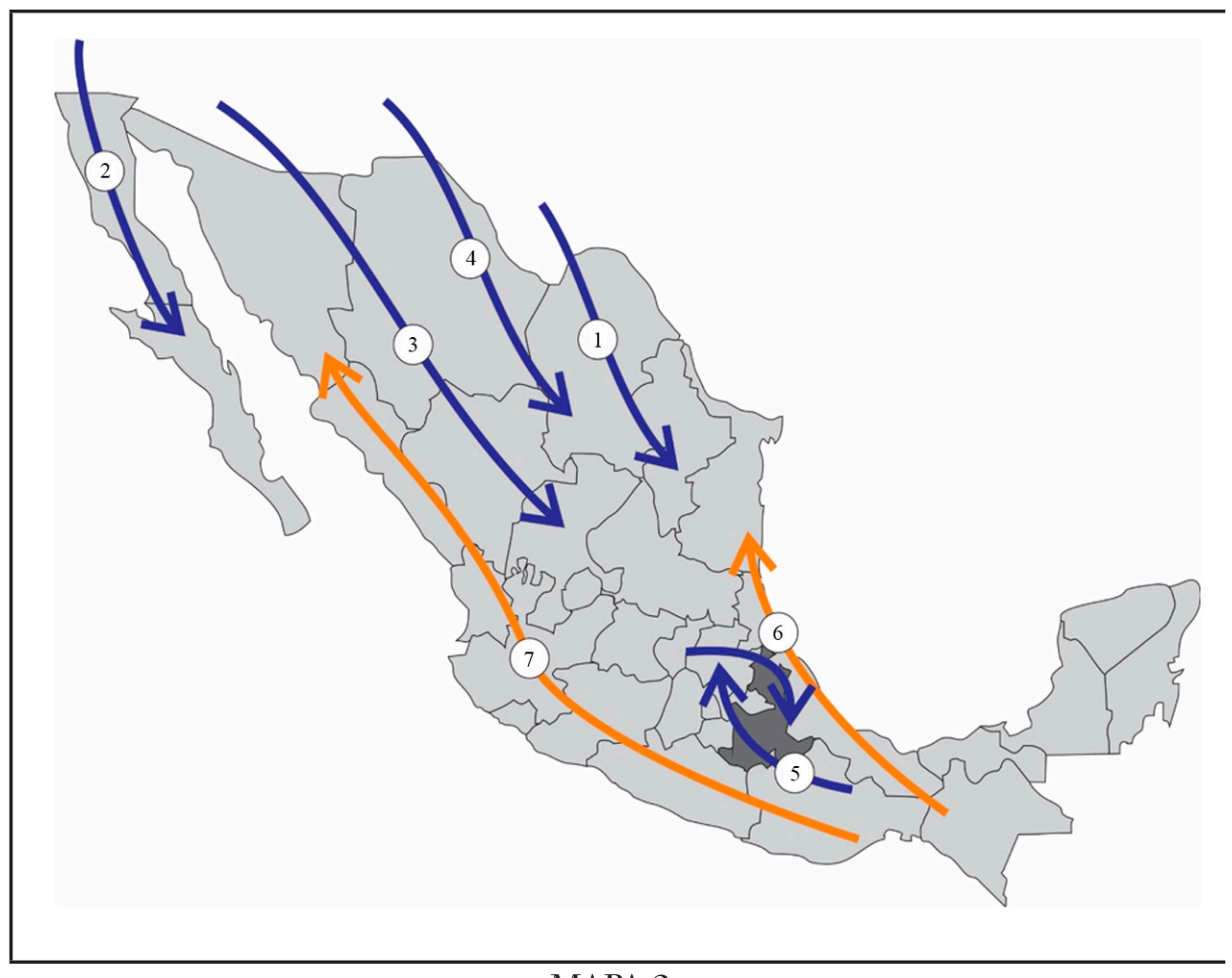

MAPA 2

Mapa que muestra los corredores biogeográficos durante el Pleistoceno Fuente: modificado de Ceballos et al. (2010).

Nota: corredores templados (en azul): 1) Este de Estados Unidos de América-Sierra Madre Oriental, 2) Estados Unidos Oriental-Baja California, 3) Montañas Rocosas-Sierra Madre Occidental, 4) Estados Unidos Central-Norte de México, 5) Eje Neovolcánico Transversal-Sierra Madre del Sur. Corredores tropicales (en naranja): 6) Tamaulipas-Planicie Costera del Golfo y 7) Sonora-Planicie Costera del Pacífico.

La presencia humana temprana en el estado de Puebla se ha registrado en diversas localidades de edad pleistocénica de la Cuenca de Valsequillo (entre ellas Tetela, El Horno, El Mirador, Caulapan, Río Atoyac, Mina del Francés, San Francisco Totimehuacán, Tecacaxco y Hueyatlaco), que es el área donde se han documentado más restos de M. columbi en el estado (Solís Torres, 2013). Esto podría soportar la hipótesis de que los primeros pobladores se establecieron en las áreas donde abundaban los mamutes, ya que es probable que fueran una de sus fuentes primarias de recursos. Sin embargo, únicamente existe evidencia de interacción humano-Mammuthus en dos localidades. La primera es San Pedro Zacachimalpa, donde la interacción está sustentada en evidencia indirecta, que consiste en una raedera de pedernal asociada a restos sin especificar (Pichardo del Barrio, 1960). La segunda localidad es Arenillas, en San Francisco Totimehuacán, donde la evidencia de interacción está dada por un artefacto de pedernal incrustado en el borde parasinfisial, por abajo del molar, de una mandíbula de Mammuthus (Armenta Camacho, 1978; Pichardo, 2005). No obstante, esta evidencia ha sido duramente cuestionada. En consecuencia, no existen pruebas sólidas que demuestren que los humanos tempranos interactuaron con Mammuthus en el estado de Puebla, por lo que la hipótesis del establecimiento de poblaciones humanas en zonas donde habitaban mamutes carece de sustento. Es más probable que la gran cantidad de registros de Mammuthus y humanos tempranos en la Cuenca de Valsequillo sea consecuencia del gran esfuerzo de muestreo, producto del trabajo de arqueólogos y paleontólogos interesados en estudiar esa zona durante varios años (algo de lo que adolecen otras localidades en el estado), y además no representan en realidad indicios de correlación humano-Mammuthus.

Arroyo-Cabrales et al. (2003b) mencionan que existen 271 localidades con restos de Mammuthus en México, de las cuales 18 están en el estado de Puebla. El recuento y adición de localidades presentadas en este trabajo 
incrementa a más del doble el número de sitios con restos de Mammuthus para Puebla. Las 38 localidades poblanas representan 14\% del total de las localidades reportadas para México y lo ubican como el segundo estado con más restos de Mammuthus en el país, sólo detrás del Estado de México, que tiene 77 (Arroyo-Cabrales et al., 2003b).

La paleodistribución de M. columbi en el estado de Puebla parece estar estrechamente ligada al paleoambiente, que a su vez tiene relación con el paleoclima presente en el Pleistoceno. Se han realizado estudios paleoclimáticos detallados en la zona de Valsequillo que permiten tener un esbozo general del paleoclima presente en áreas aledañas o con condiciones orográficas similares en Puebla. La reconstrucción paleoclimática de la Cuenca de Valsequillo, usando isótopos estables de $\delta^{18} \mathrm{O}$ y $\delta^{13} \mathrm{C}$ extraído de conchas de gasterópodos terrestres y dulceacuícolas fósiles recolectados en Barranca Caulapan, muestra que hace 35000 años el clima en esa región de Puebla era muy similar al actual; entre los 35000 y 20000 años se incrementaron las condiciones húmedas y después de los 20000 años se vuelve a elevar la humedad (Stevens et al., 2012). Aunque no existen evidencias directas, es probable que estas condiciones paleoclimáticas estuvieran presentes en gran parte del territorio poblano, principalmente en los valles centrales y regiones aledañas, favoreciendo la expansión de plantas herbáceas y el desarrollo de bosques de coníferas en los valles formados entre las zonas montañosas de Puebla. Este tipo de paleoambiente también es apoyado por la presencia en Valsequillo del perrito de la pradera Cynomys mexicanus y el conejo de zacatonal Romerolagus diazi (Cruz-Muñoz et al., 2009), especies que prefieren hábitats abiertos dominados por plantas herbáceas y pastos (Ceballos-G. y Wilson, 1985; Cervantes et al., 1990). Otro proxy que señala un ambiente abierto con presencia de pastos en la Cuenca de Valsequillo es la señal de mesodesgaste encontrada en ejemplares de E. conversidens, especie de caballo que se encuentra asociada a M. columbi en las tres zonas fosilíferas (Pichardo, 1997). La señal de mesodesgaste de E. conversidens demuestra que tenía una dieta altamente abrasiva, que sugiere ingesta de fitolitos, por lo que es probable que las poblaciones de esta especie en el área de Valsequillo tuvieran preferencia por un hábitat abierto dominado por pastos (Jiménez Moreno et al., 2016).

Los ambientes abiertos donde predomina la vegetación herbácea y que están presentes en las cuencas debieron servir como base para que las poblaciones de M. columbi se expandieran en esas zonas. La reconstrucción de la dieta de individuos de M. columbi provenientes de Valsequillo, Chalchicomula de Sesma y Amozoc, basada en el mesodesgaste de las láminas en uso de los molares y en isótopos de carbono y oxígeno obtenidos de esmalte dental, muestra que consumían principalmente plantas C4 (Pérez-Crespo et al., 2014; Carbot-Chanona et al., 2017). No obstante, estudios realizados en muestras dentales de ejemplares de $M$. columbi de otras localidades arrojan resultados que indican una dieta mixta, con ingesta de plantas C3/C4 (Pérez-Crespo et al., 2009, 2010). Esto deja de manifiesto que $M$. columbi tenía plasticidad dietaría y es probable que esta flexibilidad le haya permitido a las poblaciones encontradas en Puebla soportar la expansión y retroceso de los pastizales ocasionado por las fluctuaciones climáticas ocurridas al final del Pleistoceno. Lo anterior también ha sido discutido por Pérez-Crespo et al. (2013), quienes mencionan que individuos de M. columbi de la localidad Laguna de las Cruces, San Luis Potosí, tenían variación en su dieta ligada a los cambios ambientales.

Se ha planteado que los mamutes, al igual que los elefantes africanos actuales, se desplazaban grandes distancias en busca de agua y alimento (Haynes, 1991), por lo que otra posible hipótesis es que las poblaciones de $M$. columbi presentes en el territorio poblano migraran hacia zonas con condiciones más favorables donde predominaran los pastos, base de su dieta.

\section{Prospectiva}

Según Arroyo-Cabrales et al. (2002), Puebla cuenta con 57 localidades de edad pleistocénica, en las cuales existen evidencias de mega y mesofauna, como félidos dientes de sable, lobos, osos, mamutes, gonfoterios, camellos, llamas, berrendos, bisontes, pecaríes, tapires, caballos, perezosos gigantes y gliptodontes (Jiménez-Moreno et al., 2015; Jiménez-Hidalgo et al., 2015), por contar sólo algunos. Una de las principales áreas con restos 
de esta fauna es Valsequillo, en la cual se han centrado estudios de diversa índole desde mediados del siglo XX (Irwin-Williams, 1967; Silva-Bárcenas, 1969; Armenta Camacho, 1978). No obstante, a pesar de que Valsequillo ha sido ampliamente estudiado, el resto del estado de Puebla se ha explorado poco y, por ende, son escasos los proyectos enfocados a conocer el pasado biológico en otras partes del territorio poblano. Sin embargo, con este artículo dejamos de manifiesto la amplia distribución que tuvo M. columbi en Puebla, sobre todo en el centro, y algunas zonas del norte y sur del estado. Esto demuestra el potencial paleontológico con el que cuenta Puebla y da un punto de partida para futuras exploraciones en pro de ampliar el conocimiento sobre el pasado de México.

\section{Conclusiones}

La fauna de finales del Pleistoceno encontrada en el estado de Puebla ha sido bien estudiada, en especial la que procede del área de Valsequillo, pues se han reportado varias especies de carnívoros y herbívoros de tallas grandes, medianas y pequeñas. Una de estas especies es el mamute Mammuthus columbi, que tuvo una amplia distribución en el territorio poblano tal como lo demuestra el recuento de localidades ya reportadas y de las localidades inéditas hasta el momento que aquí se mencionan. Lo anterior posiciona a Puebla como uno de los estados con más registros de esta especie en México.

La paleodistribución de $M$. columbi al parecer está ligada a paleoambientes templados, con relativa baja humedad, ubicados entre los 1500 a 2500 msnm donde predominó la vegetación abierta. La mayoría de las localidades se ubican en la porción central de Puebla, que corresponde al corredor biogeográfico Eje Neovolcánico Transversal-Sierra Madre del Sur. La amplia distribución de M. columbi en el estado deja de manifiesto que existe un amplio potencial para ubicar más yacimientos con restos de esta u otras especies. Lo anterior abre la posibilidad de gestionar proyectos para el rescate, estudio y resguardo de nuevos restos fósiles en pro de ampliar el conocimiento biológico que se tiene hasta ahora en México.

\section{Agradecimientos}

Queremos agradecer a las autoridades del Centro INAH-Puebla, en especial a su exdelegado José Francisco Ortiz Pedraza y a Delia Domínguez Cuanalo, por las facilidades otorgadas para realizar los estudios pertinentes en el Laboratorio de Paleontología. De igual manera, a Judith Romero Rosas y Raymundo F. Rodríguez Chávez por el apoyo y amabilidad brindado para facilitar la revisión de los materiales ahí depositados. Francisco Javier Jiménez Moreno agradece al comité tutorial de la maestría en Ciencias Ambientales de la BUAP, a Ernesto Mangas, Víctor Tamariz, Constantino Gil, Fernando Aldana y Eduardo Torres por su apoyo académico y a Conacyt por la beca de posgrado. Agradecemos de manera especial a Joaquín Arroyo Cabrales del laboratorio de arqueozoología del INAH y a José Alberto Cruz Silva, Carlos Castañeda Posadas y Miguel Sánchez Salinas de la BUAP por los datos proporcionados para revisión de las localidades e identificación de ejemplares alojado en colecciones y a Jorge Aldama Peña ${ }^{\dagger}$ por el apoyo con la ubicación de las localidades en el mapa. Por último, agradecemos los comentarios de los revisores de la revista que ayudaron a mejorar sustancialmente este trabajo.

\section{ReFERENCIAS}

Armenta Camacho, J. (1978). Vestigios de labor bumana en huesos de animales extintos de Valsequillo, Puebla, México. Puebla: Publicaciones del Consejo Editorial del Gobierno del Estado de Puebla.

Arroyo-Cabrales, J., Polaco, O. J. y Johnson, E. (2002). La mastofauna del Cuaternario tardío en México, en M. Montellano Ballesteros y J. Arroyo Cabrales (Coords.), Avances en los estudios paleomastozoológicos (pp. 103-123). México: Serie Arqueológica, Instituto Nacional de Antropología e Historia. 
Arroyo-Cabrales, J., Polaco, O. J., \& Aguilar-Arellano, F. (2003a). Remains of the Mammuthus housed in the collections of the Instituto Nacional de Antropologia e Historia, Mexico. In J. W. F. Reumer, J. De Vos, \& D. Mol (Eds.), Advances in Mammoth Research (Proceedings of the Second International Mammoth Conference, Rotterdam, May 16-20, 1999). Deinsea, 9, 17-25.

Arroyo-Cabrales, J., Polaco, O. J., Johnson, E., \& Guzmán, A. F. (2003b). The distribution of the genus Mammuthus in Mexico. In J. W. F. Reumer, J. De Vos, \& D. Mol (Eds.), Advances in Mammoth Research (Proceedings of the Second International Mammoth Conference, Rotterdam, May 16-20, 1999). Deinsea, 9, 27-39.

Arroyo-Cabrales, J., Polaco, O. J., \& Johnson, E. (2007a). An overview of the Quaternary mammals from México. Courier Forschungsinstitut Senckenberg, 259, 191-203.

Arroyo-Cabrales, J., Polaco, O. J., Laurito, C., Johnson, E., Alberdi, M. T., \& Valerio Zamora, A. L. (2007b). The proboscideans (Mammalia) from Mesoamerica. Quaternary International, 169-170, $17-23$.

Arroyo-Cabrales, J., Polaco, O. J., Johnson, E., \& Ferrusquia-Villafranca, I. (2010). A perspective on mammal biodiversity and zoogeography in the Late Pleistocene of Mexico. Quaternary International, 212(2), 187-197.

Bell, C. J., Lundelius Jr., E. L., Barnosky, A. D., Graham, R. W., Lindsay, E. H., Ruez Jr.,... Zakrzewsky, R. J. (2004). The Blancan, Irvingtonian and Rancholabrean Mammal Ages. In M. O. Woodburne (Ed.), Late Cretaceous and Cenozoic mammals of North America: Biostratigraphy and Geocronology (pp. 232-314). New York: Columbia University Press.

Bravo-Cuevas, V. M., Morales-García, N. M., \& Cabral-Perdomo, M. A. (2015). Description of mastodons (Mammut americanum) from the late Pleistocene of southeastern Hidalgo, central Mexico. Boletin de la Sociedad Geológica Mexicana, 67(2), 337-347.

Carbot-Chanona, G., Lagunas Rodríguez, Z., Jiménez-Moreno, F. J. y Suárez, S. (2017). Aspectos paleobiológicos de dos ejemplares de Mammuthus columbi (Mammalia, Proboscidea, Elephantidae) del Pleistoceno de Puebla, centro de México. Boletín de la Sociedad Geológica Mexicana, 69(3), 591609.

Ceballos-G., G., \& Wilson, D. E. (1985). Cynomys mexicanus. Mammalian Species, 248, 1-3.

Ceballos, G., Arroyo-Cabrales, J., \& Ponce, E. (2010). Effects of Pleistocene environmental changes on the distribution and community structure of the mammalian fauna of Mexico. Quaternary Research, 73, 464-473.

Cervantes, F. A., Lorenzo, C., \& Hoffman, R. S. (1990). Romerolagus diazi. Mammalian Species, $360,1-7$.

Cruz Muñoz, V. (2001). Catálogo de vertebrados fósiles del Centro Regional INAH de Puebla (tesis de licenciatura). México: Benemérita Universidad Autónoma de Puebla.

Cruz-Muñoz, V., Arroyo-Cabrales, J., \& Graham, R. W. (2009). Rodents and lagomorphs (Mammalia) from the Late-Pleistocene deposits at Valsequillo, Puebla, México. Current Research in the Pleistocene, 26, 147-149.

Felix, J., \& Lenk, H. (1891). Uebersieht über die geologischen Verhältnisse des mexicanischen Staates Puebla. Beitrage zur Geologie und Paleontologie der Republik Mexico. Palaeontographica, 37, 117-139.

Freudenberg, W. (1922). Die säugetierfauna del Pliocäns und Postpliocäns von Mexico, II. Teil; Mastodonten und Elefanten. Geologische und Palaeontologische Abhandlungen, 14(3), 103-176.

García, L. D. (2014, octubre 4). Cuautinchán necesita de difusión para el turismo. Milenio. Consultado el 10 de enero de 2017. Disponible en http://www.milenio.com/region/Cuautinchan-necesita-difusion-turismo_0_278372188.html 
Gómez-Pérez, L. E. y Carbot-Chanona, G. (2012). Contribución al estudio de los megamamíferos del Pleistoceno Tardío del municipio de Villaflores, Chiapas, México. Lacandonia, 6(1), 31-41.

Graham, R. W. (1998). The Pleistocene terrestrial mammal fauna of North America. In C. M. Janis, K. M. Scott, \& L. L. Jacobs (Eds.). Evolution of Tertiary mammal of North America (pp. 66-71). United Kingdom: Cambridge University Press.

Guenther, E. W. (1968). Untersuchungen zur jungeiszeitlichen und nacheiszeitlichen geologischen und palaontologisc Geschichte. In F. Tichy (Ed.), Das Mexiko projekt der Deutschen Fors-chungsgemeinschaft-Berichte uber begonnene und geplante arbeiten (pp. 32-37). Franz Steiner Verlage GMBH-Wiesbaden.

Guenther, E. W. y Bunde, H. (1973). Investigaciones geológicas y paleontológicas en México durante los años de 1965 a 1969. Comunicaciones Proyecto Puebla-Tlaxcala, 7, 19-20.

Haynes, G. (1991). Mammoths, Mastodonts and Elephants. Biology, Behavior and the Fossil Record. New York: Cambridge University Press.

Irwin-Williams, C. (1967). Associations of early man with horse, camel, and mastodon at Hueyatlaco, Valsequillo (Puebla, Mexico). In P. S. Martin, \& H. E. Wright Jr. (Eds.), Pleistocene extinctions: the search for a cause (pp. 337-347). New Haven and London, Yale University Press.

Jiménez-Hidalgo, E., Carbot-Chanona, G., \& Castañeda Posadas, C. (2015). El registro de mamíferos fósiles de Puebla, en C. Castañeda Posadas (Coord.), El registro paleobiológico del Estado de Puebla (pp. 173-186). Puebla: Benemérita Universidad Autónoma de Puebla.

Jiménez Moreno, F. J., Carbot-Chanona, G., \& Guzmán Gutiérrez, J. R. (2015). Megafauna del Pleistoceno en Puebla. Elementos, 99, 35-39.

Jiménez Moreno, F. J., Mangas-Ramírez, E., Carbot-Chanona, G., Tamariz Flores, V., Gil Juárez, C., \& Hernández Aldana, F. (2016). Inferencia paleoambiental, con base en el análisis de mesodesgaste de tres especies fósiles del Pleistoceno, del sitio Ramsar, Valsequillo, Puebla, en III Simposio de Paleontología en el Sureste de México (pp. 56). Puebla: Benemérita Universidad Autónoma de México.

Kurtén, B., \& Anderson, E. (1980). Pleistocene mammals of North America. New York: Columbia University Press.

Lagunas Rodríguez, Z. y Suárez Cruz, S. (1997). Los restos de mamut encontrados en Tres Cerritos, Puebla. Revista Mexicana de Estudios Antropológicos, 43, 91-108.

Lagunas Rodríguez, Z., Suárez Cruz, S., \& Ocaña del Río, B. (2013). Fósiles de proboscidios en el estado de Puebla. Dualidad, 13, 24-27.

Laurito, C., \& Aguilar, D. H. (2007). El registro de Mammuthus (Proboscidea, Elephantidae) en la República de El Salvador, América Central. Revista Geológica de América Central, 34, 73-81.

Lister, A. M., \& Sher, A. V. (2015). Evolution and dispersal of mammoths across the Northern Hemisphere. Science, 350(6262), 805-809.

Lundelius, E. L., Jr. Downs, T., Lindsay, E. H, Semken, H. A., Zakrzewski, R., Churcher, C. S.,... Webb, S. D. (1987). The North American Quaternary sequence. In M. O. Woodburne (Ed.), Cenozoic Mammals of North America: Geochronology and Biostratigraphy (pp. 211-235). Berkeley: University of California Press.

Montellano-Ballesteros, M. (2000). The Quaternary mammals from the Axamilpa River. Guide Book of the Field Trips, 60nd Annual Meeting of Society Vertebrate Paleontology. Avances en Investigación. Publicación Especial de la Universidad Autónoma de Hidalgo, 120-123.

Ogg, J. G., Ogg, G. M., \& Gradstein, F. M. (2016). A concise geologic time scale. Amsterdam: Elsevier.

Olsen, S. J. (1972). The American mastodon and the woolly mammoth. Osteology for the archaeologist. Papers of the Peabody Museum of Arcabeology and Ethnology, Harvard University, 56(3), 1-43. 
Osborn, H. F. (1905). Recent vertebrate paleontology fossil mamals of Mexico. Sciencie, 21(546), 931-932.

Osborn, H. F. (1942). Proboscidea: a monograph of the discovery, evolution, migration, and extinction of the mastodons and elephants of the world. Vol. II: Stegodontoidea, Elephantoidea. New York: The American Museum Press.

Pérez-Crespo, V. A., Arroyo-Cabrales, J., Benammi, M., Santos-Moreno, A., Morett-A., L., Morales-Puente, P., \& Cienfuegos-Alvarado, E. (2010). Carbon isotopic values of tooth enamel of Mammuthus columbi from Tocuila, State of Mexico, Mexico. Current Research in the Pleistocene, 27, 188-190.

Pérez-Crespo, V. A., Arroyo-Cabrales, J., Alva-Valdivia, L. M., Morales-Puente, P., Cienfuegos-Alvarado, E., Otero, F. J. y Ochoa-Castillo, P. (2014). La paleodieta de cinco especies de mamíferos herbívoros Rancholabreanos de Valsequillo (Puebla, México). Revista Chilena de Antropología, 30, 76-82.

Pérez-Crespo, V. A., Arroyo-Cabrales, J., Benammi, M., Polaco, O. J., Santos-Moreno, A., Morales-Puente,... Otero, F. J. (2013). Variación de la dieta del mamut de las praderas de Laguna de las Cruces, San Luis Potosí, México. Boletín de la Sociedad Geológica Mexicana, 65(3), 573-580.

Pérez-Crespo, V. A., Sánchez-Chillón, B., Arroyo-Cabrales, J., Alberdi, M. A., Polaco, O. J., Santos-Moreno, A.,... Cienfuegos-Alvarado, E. (2009). La dieta y el hábitat del mamut y los caballos del Pleistoceno tardío de El Cedral con base en isótopos estables ( $\delta 13 \mathrm{C}, \delta 18 \mathrm{O})$. Revista Mexicana de Ciencias Geológicas, 26(2), 347-355.

Pichardo, M. (1997). Valsequillo biostratigraphy: New evidence for Pre-Clovis date. Anthropologischer Anzeiger, 55(3/4), 233-246.

Pichardo, M. (2005). Taxonomic revision of central Mexican mammoths in paleoindian sites. Anthropologischer Anzeiger, Jg., 63, 409-413.

Pichardo del Barrio, M. (1960). Proboscídeos fósiles de México: una revisión. Instituto Nacional de Antropología e Historia, Colección Cientifica, 4, 1-63.

Silva-Bárcenas, S. A. (1969). Localidades de vertebrados fósiles en la República Mexicana. Paleontología Mexicana, 28, 1-34.

Solís Torres, Ó. R. (2013). Estudio de los restos óseos de fauna del Pleistoceno recuperados en la excavación arqueológica de Hueyatlaco, temporadas de campo 2001-2004. Escuela Nacional de Antropología e Historia. México.

Stevens, R. E., Metcalfe, S. E., Leng, M. J., Lamb, A. L., Sloane, H. J., Naranjo, E., \& Silvia González, S. (2012). Reconstruction of late Pleistocene climate in the Valsequillo Basin (Central Mexico) through isotopic analysis of terrestrial and freshwater snails. Palaeogeography, Palaeoclimatology, Palaeoecology, 319-320, 16-27.

Suárez Cruz, S. y Lagunas Rodríguez, Z. (2008). Los restos de mamut de Santa Catarina Villa Nueva, Puebla, en C. Serrano Sánchez y M. A. Cardoso Gómez (Eds.), El Mediterráneo Americano: población, cultura e Historia, 1 (pp. 469-476). XXVII Mesa Redonda, Sociedad Mexicana de Antropología/ Instituto de Investigaciones Antropológicas, México: Universidad Nacional Autónoma de México.

Tapia-Ramírez, G., Guzmán, A. F. y Polaco, O. J. (2013). Los gonfoterios (Proboscidea, Gomphotheriidae) de Colima, México. Boletín de la Sociedad Geológica Mexicana, 65(3), 591-607.

Torquemada, J. (1969). Monarquia Indiana. Tomo 1. México: Porrúa.

Torres-Martínez, A., \& Agenbroad, L. D. (1991). Preliminary report of the Pleistocene mammals of the Valley of the Axamilpa River, near Tepeji de Rodríguez, Puebla, México. Current Research in the Pleistocene, 8, 99-102.

Tovar, R. E., Sedov, S., Montellano-Ballesteros, M., \& Solleiro, E. (2014). The paleoecological interpretation of Santa Cruz Nuevo, Mexico, based on fossil vertebrates and alluvial pedosediments during MIS 3. Revista Mexicana de Ciencias Geológicas, 31(3), 407-419. 
ANEXO 1

Restos de Mammuthus columbi del estado de Puebla depositado en colecciones mexicanas

\begin{tabular}{|c|c|c|}
\hline Número de catálogo & Tipo de material & Localidad \\
\hline CRINAHP 0065 & M2 derecho con cinco láminas & $\mathrm{s} / 1$ \\
\hline CRINAHP 0066 & Molar inferior fragmentado con siete láminas conservadas & $\mathrm{s} / 1$ \\
\hline CRINAHP 0067 & Molar superior fragmentado con cinco láminas conservadas & $\mathrm{s} / 1$ \\
\hline CRINAHP 0288 & Molar superior izquierdo con cinco láminas conservadas & $\mathrm{s} / 1$ \\
\hline CRINAHP 0289 & Molar inferior fragmentado con ocho láminas conservadas & $\mathrm{s} / 1$ \\
\hline CRINAHP 0296 & Molar superior derecho con siete láminas conservadas & $\mathrm{s} / 1$ \\
\hline CRINAHP 0072 & Molar inferior derecho cuatro láminas conservadas & $\mathrm{s} / 1$ \\
\hline CRINAHP 0495 & Vértebra & Caulapan \\
\hline CRINAHP 0506 & $\begin{array}{l}\text { Fragmento de molar inferior izquierdo con nueve láminas } \\
\text { conservadas }\end{array}$ & $\mathrm{s} / 1$ \\
\hline CRINAHP 0507 & $\begin{array}{l}\text { Fragmento de molar inferior derecho con siete láminas } \\
\text { conservadas }\end{array}$ & $\mathrm{s} / 1$ \\
\hline CRINAHP 0496 & Centro de vértebra torácica & Caulapan \\
\hline CRINAHP 0629 & $\begin{array}{l}\text { Fragmento posterior de molar superior derecho con siete } \\
\text { láminas conservadas }\end{array}$ & Ixtacamaxtitlán \\
\hline CRINAHP 0637 & Molar fragmentado con cuatro láminas & $\begin{array}{l}5 \text { de Mayo y } 2 \text { poniente, Centro } \\
\text { Histórico }\end{array}$ \\
\hline CRINAHP 0714 & Vértebra lumbar sin apófisis espinosa & Zacachimalpa \\
\hline CRINAHP 0645 & Atlas & Valsequillo \\
\hline CRINAHP 1044 & Molar fragmentado con siete láminas conservadas & $\mathrm{s} / 1$ \\
\hline CRINAHP 1045 & Molar superior izquierdo con once láminas conservadas & $\mathrm{s} / 1$ \\
\hline CRINAHP 1046 & Molar con ocho láminas conservadas & $\mathrm{s} / 1$ \\
\hline CRINAHP 1645 & Fragmento de mandíbula con molar & Ocotitlán \\
\hline CRINAHP 1296 & Lámina aislada & Arenillas \\
\hline CRINAHP 1302 & Molar fragmentado mal preservado & Arenillas \\
\hline CRINAHP 1774 & Fragmento de molar con dos láminas & $\mathrm{s} / 1$ \\
\hline CRINAHP 1777 & Fragmento de molar con siete láminas & $\mathrm{s} / 1$ \\
\hline CRINAHP 1778 & Fragmento de molar con cuatro láminas & $\mathrm{s} / 1$ \\
\hline CRINAHP 1310 & Molar fragmentado & Arenillas \\
\hline CRINAHP 2123 & Fragmento de defensa & Tres Cerritos \\
\hline CRINAHP 2124 & Fragmento de escápula & Tres Cerritos \\
\hline CRINAHP 2126 & Fragmento de escápula & Tres Cerritos \\
\hline CRINAHP 2134 & Fragmento de defensa & Lagunillas del Rayón \\
\hline CRINAHP 2171 & Húmero fragmentado & Nopalucan \\
\hline CRINAHP 2172 & Fragmento del cráneo & San Francisco Totimehuacán \\
\hline CRINAHP 2274 & $\begin{array}{l}\text { Cráneo parcial con el M2 derecho, el M2 y M3 izquierdos } \\
\text { y parte de la defensa izquierda }\end{array}$ & Sierra de Amozoc \\
\hline CRINAHP 2275 & $\begin{array}{l}\text { Ambos M2 y M3, fémur derecho al cual le falta el extremo } \\
\text { proximal, tibia parcialmente conservada, vértebras incom- } \\
\text { pletas y varios fragmentos de costillas }\end{array}$ & Barranca El Morillo \\
\hline CRINAHP 2284 & m3 izquierdo con ocho láminas & $\mathrm{s} / \mathrm{l}$, pero se presume Valsequillo \\
\hline CRINAHP 2286 & m3 aislado & $\mathrm{s} / \mathrm{l}$, pero se presume Valsequillo \\
\hline CRINAHP 2287 & m1 aislado & $\mathrm{s} / \mathrm{l}$, pero se presume Valsequillo \\
\hline
\end{tabular}


ANEXO 1 (continúa)

\begin{tabular}{|c|c|c|}
\hline CRINAHP 2296 & M1 aislado & s/l, pero se presume Valsequillo \\
\hline CRINAHP 2297 & $\mathrm{~m} 2$ aislado & $\mathrm{s} / \mathrm{l}$, pero se presume Valsequillo \\
\hline CRINAHP 2298 & m3 fragmentado con diez láminas conservadas & $\mathrm{s} / \mathrm{l}$ pero se presume Valsequillo \\
\hline CRINAHP 2299 & M3 fragmentado & $\mathrm{s} / \mathrm{l}$ pero se presume Valsequillo \\
\hline BUAPPALZ 0004 & Parte distal del húmero & Valsequillo \\
\hline BUAPPALZ 0025 & Alveolos de los incisivos & Atexcal \\
\hline BUAPPALZ 0026 & Alveolos de los incisivos & Atexcal \\
\hline BUAPPALZ 0033 & Maxilar & Valsequillo \\
\hline BUAPPALZ 0055 & Fragmento de cráneo & Atexcal \\
\hline BUAPPALZ 0056 & Fragmento de cráneo & Atexcal \\
\hline BUAPPALZ 0057 & Fragmento de cráneo & Atexcal \\
\hline BUAPPALZ 0060 & Fragmento del arco zigomático & Atexcal \\
\hline BUAPPALZ 0079 & Maxilar & Valsequillo \\
\hline BUAPPALZ 0081 & Fragmento distal del fémur & Valsequillo \\
\hline BUAPPALZ 0082 & Fémur & Valsequillo \\
\hline BUAPPALZ 0083 & Cóndilo articular del fémur & Valsequillo \\
\hline BUAPPALZ 0085 & Fémur fragmentado & Atexcal \\
\hline BUAPPALZ 0092 & Parte distal del húmero & Valsequillo \\
\hline BUAPPALZ 0102 & Acetábulo & Atexcal \\
\hline BUAPPALZ 0109 & Parte distal de la tibia & Valsequillo \\
\hline BUAPPALZ 0110 & Parte distal del húmero & Valsequillo \\
\hline BUAPPALZ 0114 & Molar aislado & Atexcal \\
\hline BUAPPALZ 0115 & Molar aislado & Atexcal \\
\hline BUAPPALZ 0116 & Molar aislado & Atexcal \\
\hline BUAPPALZ 0117 & Molar aislado & Atexcal \\
\hline BUAPPALZ 0118 & Molar aislado & Atexcal \\
\hline BUAPPALZ 0119 & Molar aislado & Atexcal \\
\hline BUAPPALZ 0969 & Vértebra lumbar & Valsequillo \\
\hline BUAPPALZ 0997 & Fragmento de vértebra & Valsequillo \\
\hline BUAPPALZ 1012 & Molar inferior aislado & Valsequillo \\
\hline BUAPPALZ 1061 & Vértebra lumbar & Valsequillo \\
\hline Sin número* & Fragmento de molar inferior & $\begin{array}{l}\text { Cerro Chiquihuite, San Francisco } \\
\text { Totimehuacán }\end{array}$ \\
\hline Sin número** & Cráneo con molares & Cuautinchán \\
\hline
\end{tabular}

Fuente: elaboración propia.

Nota: * este ejemplar está bajo resguardo del grupo Tepalcayotl; ${ }^{* *}$ este ejemplar se encuentra resguardado en el exconvento de San Juan Bautista y carece de número de colección. CRINAHP = Colección Regional Instituto Nacional de Antropología e Historia, Puebla; BUAPPALZ = Colección Paleontológica, sección Vertebrados, Benemérita Universidad Autónoma de Puebla.

\section{BY-NC-ND}

